\title{
A modified semi-selective medium for isolation and enumeration of Pochonia chlamydosporia (Goddard) Zare \& W. Gams
}

\author{
M K Rincy ${ }^{1,2}$, R Praveena ${ }^{1 *}$, S J Eapen ${ }^{1}$ \\ ${ }^{1}$ Division of Crop Protection, ICAR-Indian Institute of Spices Research, Kozhikode - 673 012, Kerala, India \\ ${ }^{2}$ University of Calicut, Malappuram - 673635, Kerala, India \\ *E-mail: Praveena.R@icar.gov.in
}

Received 24 February 2021; Revised 11 June 2021; Accepted 22 June 2021

\begin{abstract}
Pochonia chlamydosporia, is one of the most promising biological control agents for managing phytoparasitic nematodes. Isolation and enumeration of viable colonies of $P$. chlamydosporia from soil and other substrates without contamination is a major limitation, when commonly available nutrient media are used. Development of a suitable selective/semi-selective media by incorporating one or more inhibitors of microbial growth can facilitate isolation of the fungus. In vitro studies were carried out to test the compatibility of commonly used pesticides, namely, metalaxyl, metalaxyl-mancozeb, carbendazim, copper oxychloride, and chlorpyrifos with P. chlamydosporia. The fungus showed relatively high tolerance to higher doses of metalaxyl and carbendazim and was used in the modified medium for better suppression of other soil borne fungi. In the present study, Kerry's semi-selective medium was modified and evaluated by counting the viable fungal propagules in different substrates (rice, farmyard manure, maize, rice bran, barley, and sorghum) and soil artificially inoculated with the fungus. The results showed that the modified Kerry's semi-selective medium can effectively be used for isolation and quantification of $P$. chlamydosporia in routine studies.
\end{abstract}

Keywords: biological control, Pochonia chlamydosporia, quantification, semi-selective medium.

\section{Introduction}

Currently, phytoparasitic nematode management strategies are mainly based on nematicides, host plant resistance and crop rotation. Intensive use of nematicides results in critical environmental hazards (Aravind et al. 2009). The need for alternatives to chemical nematicides has accelerated research on eco-friendly measures to manage parasitic nematodes (Fourie et al. 2016). Biological control is an alternative management technique that can minimize the population of nematodes directly through parasitism or indirectly through the production of toxic metabolites and thus can address the possible environmental problems associated with chemical control of phytoparasitic nematodes (Dong \& Zhang 2006). 
Pochonia chlamydosporia (Goddard) Gams \& Zare, a ubiquitous facultative hyperparasitic fungus, is one the most promising biocontrol agents recommended for the management of phytoparasitic nematodes. The fungus is known to infect economically significant nematode genera like Meloidogyne, Globodera, and Heterodera (Esteves et al. 2009). The fungus is a facultative parasite capable of surviving in soil as a saprotroph and parasitizing mainly the eggs and females of nematodes (Siddiqui et al. 2009). The fungus usually parasitizes nematode eggs without producing any specialized structures. There are various life stages of $P$. chlamydosporia, which comprise of hyphae with different nucleus numbers as well as unicellular conidiospores and multicellular chlamydospores (Kerry 2000). Chlamydospores produced by the fungus allow it to withstand challenging environmental conditions such as drought or cold. P. chlamydosporia strains, however, vary in their virulence, capability to colonize root surfaces, and development of chlamydospore, as well as in their effectiveness to control nematode populations (Bourne et al. 1994; Morton et al. 2003; Mauchline et al. 2004). To understand the efficiency of $P$. chlamydosporia, it is important to monitor the growth, establishment and survival in the soil and on plant roots. Quantification of $P$. chlamydosporia is a limitation, since the fungus does not consist of propagules that are of same size and genetic material (Manzanilla-lópez et al. 2011). Besides, quantitative estimation of the fungal population from rhizosphere soil samples is often difficult because of the slow growing nature of the fungus on conventional agar media.

Isolation and counting of $P$. chlamydosporia colonies will be much easier and faster if a suitable semi-selective media is developed by incorporating one or more inhibitors of microbial growth. The usage of semi-selective media for isolation and enumeration can restrict bacterial contamination and fastgrowing fungal colonies, and can also aid in determining the comparative abundance of fungus in the root rhizosphere infected with nematodes (Mauchline et al. 2002; Atkins et al. 2003a). Enumeration of P. chlamydosporia from soil and other substrates using Kerry's semiselective medium results in the contamination of the medium by colonies of other soil borne fungi. The present study was therefore carried out to develop a modified semi-selective medium for isolation and quantification of the fungus from humid tropical soils and other substrates.

\section{Materials and Methods}

Culture characteristics of $P$. chlamydosporia (NAIMCC-SF-0048)

The isolate used in the present study was $P$. chlamydosporia (NAIMCC-SF-0048) maintained in the repository for biocontrol agents at ICARIndian Institute of Spices Research (IISR), Kozhikode, India. The identity of the strain was confirmed by DNA fingerprinting at National Buraeu of Agriculturally Important Microorganisms (NBAIM), Mau, Uttar Pradesh.

To study the culture characteristics, mycelial discs of size $5 \mathrm{~mm}$ (diameter), taken from the edges of the $P$. chlamydosporia colony were placed in the centre of the potato dextrose agar

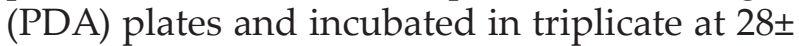
$2^{\circ} \mathrm{C}, 70-80 \% \mathrm{RH}$ and a $12: 12 \mathrm{~h}$ day: night photo phase. The mean radial mycelial growth $(\mathrm{mm})$, colony size and colour were recorded 10 days after inoculation (DAI). The experiment was repeated once to confirm the results.

Conidial morphology was studied by mounting the 14 day old fungal culture in lactophenol on microscopic slides and the size of conidia was measured at 10x and 40x magnification under a bright field microscope (Leica DM5000B). The morphological characters were compared with the descriptions given in the published keys of the fungal species (Sung et al. 2007; Atkins et al. 2003b).

In vitro compatibility of $P$. chlamydosporia with pesticides

Five pesticides viz., carbendazim (Bavistin $50 \%$ WP), metalaxyl-mancozeb (Master $72 \%$ WP), metalaxyl (Metalaxyl 35\% WP), copper oxychloride (Blitox 50\% WP), and 
chlorpyrifos (Anth 50\% EC) were tested for their compatibility with $P$. chlamydosporia by poisoned food technique (Bruin et al. 1981) at the recommended dose, two lower doses and two higher doses as given in Table 1. Stock solution of each pesticide was prepared by adding the requisite quantity of the chemical in sterile distilled water and added to molten PDA in Erlenmeyer flasks separately to get the final concentrations. The poisoned medium was poured into sterile Petri dishes of $9 \mathrm{~cm}$ diameter and allowed to cool. Mycelial discs of size $5 \mathrm{~mm}$ diameter taken from the edge of $P$. chlamydosporia colony was placed in the centre of each Petridish and incubated for 10 days as described above. Petri dishes with nonpoisoned medium served as the control. Each treatment was replicated thrice. The radial growth of the colony was measured in each treatment and the percentage inhibition of growth was determined using the formula: I $=\mathrm{C}-\mathrm{T} / \mathrm{C} \times 100$ where, $\mathrm{I}$ is percentage growth inhibition, $C$ is radial growth in control $(\mathrm{mm})$ and $\mathrm{T}$ is radial growth in treated plates $(\mathrm{mm})$. The tolerance limit of pesticide concentration for the fungus was worked out and values were statistically analysed.

\section{Modification of Kerry's semi-selective medium}

The Kerry's semi-selective medium (Kerry et al. 1993) is as follows: $17 \mathrm{~g} \mathrm{CMA}, 17.5 \mathrm{~g} \mathrm{NaCl}$, $75 \mathrm{mg}$ Rose Bengal, $37.5 \mathrm{mg}$ of carbendazim and thiabendazole, $50 \mathrm{mg}$ each of the antibiotics chloramphenicol, aureomycin and streptomycin sulphate and $3 \mathrm{ml}$ Triton X-100 per litre. In the present study, the semi-selective medium was modified with the following composition: $17 \mathrm{~g}$ corn meal agar, $17.5 \mathrm{~g} \mathrm{NaCl}$,
$75 \mathrm{mg}$ Rose Bengal, $50 \mathrm{mg}$ each of metalaxyl and carbendazim, $3 \mathrm{~mL}$ Triton X-100, $50 \mathrm{mg}$ each of rifampicin, streptomycin sulphate and chloramphenicol per litre.

\section{Evaluation of modified semi selective media}

The modified semi selective media was evaluated by dilution-plate technique. Serial dilutions were prepared from $1 \mathrm{ml}$ liquid inoculum of $P$. chlamydosporia grown in potato dextrose broth (PDB) as described above. Aliquots $(0.2 \mathrm{ml})$ of each dilution in triplicate were aseptically spread on the surface of the selective media in Petri dishes $(9 \mathrm{~cm})$ and the plates were incubated as described above for 10 days. The colonies of serially diluted plates were counted and expressed in terms of colony forming units (CFU) $\mathrm{mL}^{-1}$.

Isolation and quantification of P. chlamydosporia from different solid substrates and soil

The efficiency of the modified Kerry's semiselective medium was studied using different solid substrates namely, sorghum, rice, barley, rice bran, maize, farmyard manure, and soil artificially inoculated with $P$. chlamydosporia. For this, $40 \mathrm{~g}$ each of sorghum, rice, barley, rice bran, and maize were soaked separately in distilled water for 1-2 hours. The soaked grains were washed with distilled water and the substrates were autoclaved at 15 psi for 20 minutes. To inoculate the solid substrates, liquid inoculum of $P$. chlamydosporia was prepared by inoculating PDB in Erlenmeyer flasks with mycelial discs of size $5 \mathrm{~mm}$ taken from the margins of the colony (5 discs 250 $\mathrm{ml}^{-1}$ media). The flasks were kept inside an incubator shaker maintained at $28 \pm 2^{\circ} \mathrm{C}, 180$ rpm for 10 days.

Table1. Pesticides and concentrations used for in vitro compatibility studies with Pochonia chlamydosporia

\begin{tabular}{lccccc}
\hline Pesticide & \multicolumn{5}{c}{ Concentrations tested (ppm) } \\
\cline { 2 - 6 } & $1 / 4 \mathrm{x}$ & $1 / 2 \mathrm{x}$ & $\mathrm{x}$ & $2 \mathrm{x}$ & $4 \mathrm{x}$ \\
\hline Metalaxyl & 250 & 500 & 1000 & 2000 & 4000 \\
Carbendazim & 250 & 500 & 1000 & 2000 & 4000 \\
Metalaxyl - mancozeb & 312.5 & 625 & 1250 & 2500 & 5000 \\
Copper oxychloride & 500 & 1000 & 2000 & 4000 & 8000 \\
Chlorpyriphos & 750 & 1500 & 3000 & 6000 & 12000 \\
\hline
\end{tabular}


After sterilization, each flask with the solid substrates was inoculated with $10 \mathrm{ml}$ of the liquid fungal inoculum. The inoculated flasks were shaken at regular interval to obtain uniform growth of the fungus. The assays were carried out in triplicates and the experiment was repeated three times to confirm the results. $P$. chlamydosporia on different solid substrates and soil was quantified 14 days after inoculation. The colonies were counted and expressed in terms of CFU g ${ }^{-1}$ of the substrate.

Isolation and quantification of P. chlamydosporia from in planta studies

A pot experiment was set to test the efficacy of the developed medium for enumerating the colonies of $P$. chlamydosporia in soils under greenhouse conditions $\left(28 \pm 2{ }^{\circ} \mathrm{C}\right.$, relative humidity $70-80 \%$. One month old black pepper plants (Variety: IISR Sreekara) were planted in pots filled with potting mixture (1 $\mathrm{kg}$ ) like sterilized soil, non-sterilized soil and vermiculite + farmyard manure. Inoculum of $P$. chlamydosporia $\left(10^{8} \mathrm{cfu} \mathrm{g}^{-1}\right)$ mass multiplied on rice grains were applied to the planting media at different doses ( $1 \mathrm{~g}, 3 \mathrm{~g}$ and $5 \mathrm{~g}$ ) one month after planting. All the treatments were replicated thrice and control plants were maintained without inoculating the fungus. Soil sampling was done 1 month after post inoculation, serially diluted and plated in semiselective medium and incubated at $28 \pm 2{ }^{\circ} \mathrm{C}$, $70-80 \%$ RH for 10 days. The colonies of serially diluted plates were counted and expressed in terms of CFU $\mathrm{mL}^{-1}$.

\section{Results and Discussion}

The isolate of $P$. chlamydosporia used in the present study produced white cottony mycelium, with a light-yellow centre and even edges. The colony diameter ranged between 2.5 - $3.0 \mathrm{~cm}$ on PDA, seven days after inoculation indicating the slow growing nature of the fungus. Microscopic observation showed that the conidia were produced individually on vegetative hyphae or in two whorls on erect conidiophores. The mycelium was verticillate in nature and the conidia produced were ellipsoid in shape. The morphological characters of the isolate used in the study were in confirmation with the descriptions given by Zare et al. (2001). Based on the fingerprinting data of NBAIM Mau, the fungal isolate was confirmed as $P$. chlamydosporia, with the accession number NAIMCC-SF-0048. According to Zare et al. (2001), conidial characters are mainly used to identify the variety of $P$. chlamydosporia. The isolates of $P$. chlamydosporia var. catenulate produced conidia which are more globose to subglobose in shape whereas, P. chlamydosporia var. chlamydosporia produced ellipsoid conidia.

Due to the slow growing nature of the fungus on culture media, quantitative estimation of the population in soil and other substrates is a major limitation. For the easy isolation and identification of $P$. chlamydosporia, the use of an appropriate semi-selective media with microbial inhibitors is essential. Use of semi selective media can prevent the growth of bacteria and other fungi and also help to estimate the population of the fungus in the rhizosphere of plants (Mauchline et al. 2002; Atkins et al. 2003a). In the present study, when Kerry's semi-selective medium was used for soil population estimation, the inoculated plates got contaminated with the colonies of other soil inhabiting fungi viz., Pythium, Fusarium etc. Hence attempts were made to amend the above medium so that isolation and diagnosis of the target fungus from humid tropical soils and other substrates was easy and accurate.

The in vitro compatibility studies of $P$. chlamydosporia with the five pesticides viz., carbendazim, metalaxyl- mancozeb, metalaxyl, copper oxychloride, and chlorpyrifos clearly showed that the fungus could withstand high concentrations of metalaxyl (0.4\%) and carbendazim when compared to other pesticides (Table 2). The effective dose concentrations (ED50) of metalaxyl and carbendazim were found to be $2.36 \%$ and $1.32 \%$, respectively. In vitro effects of different concentrations of metalaxyl and carbendazim on mycelial growth of $P$. chlamydosporia are shown in Figures 1 and 2, respectively. Copper oxychloride, the combination product of 
Table 2. Compatibility of Pochonia chlamydosporia with different pesticides

\begin{tabular}{|c|c|c|c|}
\hline Pesticides & Concentration (ppm) & $\begin{array}{l}\text { Percentage growth } \\
\text { inhibition (I) }(\%)^{*}\end{array}$ & $\mathrm{ED}_{50}$ \\
\hline \multirow[t]{5}{*}{ Metalaxyl } & 250 & $4.76 \pm 0.017^{\mathrm{D}}$ & \multirow{5}{*}{2.36} \\
\hline & 500 & $9.52 \pm 0.011^{\mathrm{C}}$ & \\
\hline & 1000 & $9.52 \pm 0.029^{C}$ & \\
\hline & 2000 & $19.04 \pm 0.023^{\text {В }}$ & \\
\hline & 4000 & $23.80 \pm 0.005^{\mathrm{A}}$ & \\
\hline \multirow[t]{5}{*}{ Carbendazim } & 250 & $33.3 \pm 0.034^{\mathrm{B}}$ & \multirow{5}{*}{1.32} \\
\hline & 500 & $33.3 \pm 0.040^{\mathrm{B}}$ & \\
\hline & 1000 & $42.85 \pm 0.023^{\mathrm{A}}$ & \\
\hline & 2000 & $42.85 \pm 0.017^{\mathrm{A}}$ & \\
\hline & 4000 & $42.85 \pm 0.028^{\mathrm{A}}$ & \\
\hline \multirow[t]{5}{*}{ Metalaxyl - Mz } & 312.5 & $42.85 \pm 0.046^{\mathrm{C}}$ & \multirow{5}{*}{$0.05 \mathrm{c}$} \\
\hline & 625 & $42.85 \pm 0.080^{\mathrm{C}}$ & \\
\hline & 1250 & $52.38 \pm 0.046^{\mathrm{B}}$ & \\
\hline & 2500 & $100 \pm 0^{\mathrm{A}}$ & \\
\hline & 5000 & $100 \pm 0^{\mathrm{A}}$ & \\
\hline \multirow[t]{5}{*}{ Copper oxychloride } & 500 & $33.3 \pm 0.069^{\mathrm{B}}$ & \multirow{5}{*}{0.051} \\
\hline & 1000 & $33.3 \pm 0.086^{\mathrm{B}}$ & \\
\hline & 2000 & $100 \pm 0^{\mathrm{A}}$ & \\
\hline & 4000 & $100 \pm 0^{\mathrm{A}}$ & \\
\hline & 8000 & $100 \pm 0^{\mathrm{A}}$ & \\
\hline \multirow[t]{5}{*}{ Chlorpyriphos } & 750 & $42.8 \pm 0.057^{C}$ & \multirow{5}{*}{0.11} \\
\hline & 1500 & $47.6 \pm 0.029^{\mathrm{B}}$ & \\
\hline & 3000 & $100 \pm 0^{\mathrm{A}}$ & \\
\hline & 6000 & $100 \pm 0^{\mathrm{A}}$ & \\
\hline & 12000 & $100 \pm 0^{\mathrm{A}}$ & \\
\hline
\end{tabular}

* Values are means of three replicates \pm standard error (S.E). I = C - T/C x 100 where, $\mathrm{I}=$ Percentage growth inhibition; $C=$ Radial growth in control $(\mathrm{mm}) ; \mathrm{T}=$ Radial growth in treated plates $(\mathrm{mm}), \mathrm{ED}_{50}$ (Effective dose). ${ }^{*}$ Means followed by the same letter are not statistically different.

metalaxyl - mancozeb, and chlorpyriphos inhibited the fungus at concentrations above $2000 \mathrm{ppm}$. Based on the in vitro evaluation, the fungicides metalaxyl and carbendazim were selected to modify Kerry's semiselective medium. The compatibility studies of 14 agropesticides on the development and sporulation of nematophagous fungi showed that the isolates of Paecilomyces sp. and Pochonia sp. were less sensitive to the pesticides tested than Arthrobotrys species
(Mensinet al. 2013). Jacobs et al. (2003) reported that the nematophagous fungi such as $P$. lilacinus, Plectosphaerella cucumerina, and $P$. chlamydosporia were compatible with the chemical tolclofos-methyl.

Based on the leads obtained in the present study, a modified semi-selective medium with the following composition was prepared as explained in materials and methods. The fungicides and antibiotics used in the medium can inhibit the growth of other fungi and 


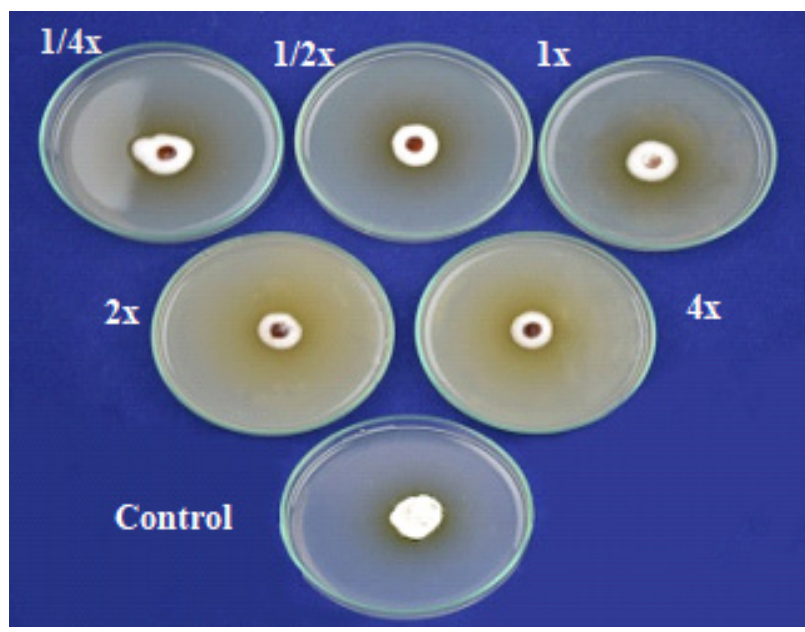

Fig 1. In vitro evaluation of different concentrations of metalaxyl on mycelial growth of Pochonia chlamydosporia (1/4x- $250 \mathrm{ppm}, 1 / 2 \mathrm{x}-500 \mathrm{ppm}$, 1x-1000 ppm, 2x- 2000 ppm, 4x- 4000 ppm)

bacteria, respectively. $\mathrm{NaCl}$ and Triton X-100 reduced the rate of colony growth and the Rose Bengal also prevented the growth of microbes. Manzanilla-lópez et al. (2017) reported that the microbial contamination in media can affect the isolation of nematophagous fungi. The use of fungicides metalaxyl and carbendazim in the modified media prevented the fastgrowing fungi like Pythium and Fusarium species, respectively, and the identification and enumeration of $P$. chlamydosporia colonies were comparatively easier on this modified medium. The reddish yellow colonies of $P$. chlamydosporia obtained after serial dilution in modified semi-selective media are shown in Figure 3. The colonies formed on semiselective media were reinoculated on potato dextrose agar to reconfirm the colony characters. The reinoculated colonies showed the morphological characters of the test isolate used for the study.

Growth of P. chlamydosporia on various solid substrates viz. sorghum, rice, barley, rice bran, maize, farmyard manure, was enumerated using the modified medium (Figure 4). Among the substrates tested, highest CFU $\mathrm{g}^{-1}$ was observed with rice grains; the population recorded was significantly higher compared

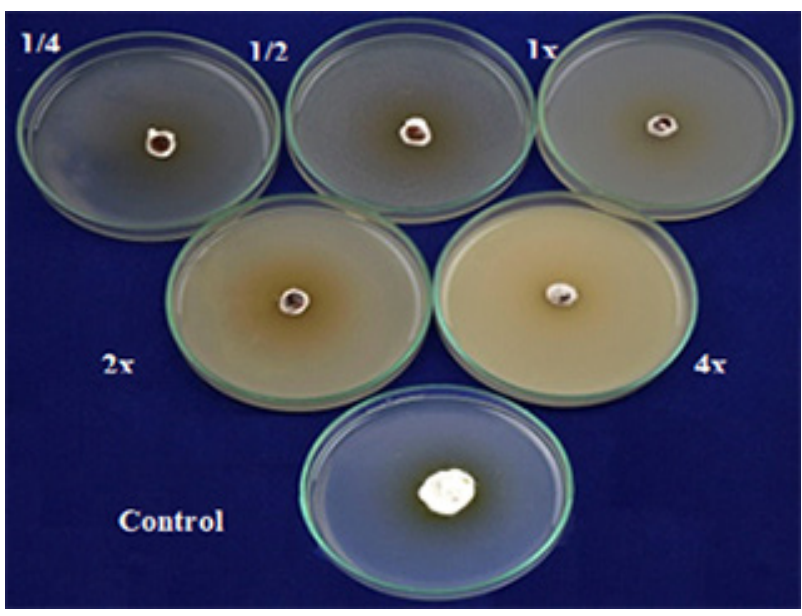

Fig 2. In vitro evaluation of different concentrations of carbendazim on mycelial growth of Pochonia chlamydosporia (1/4x- $250 \mathrm{ppm}, 1 / 2 \mathrm{x}-$ 500 ppm, 1x-1000, 2x- 2000 ppm, 4x- 4000 ppm)

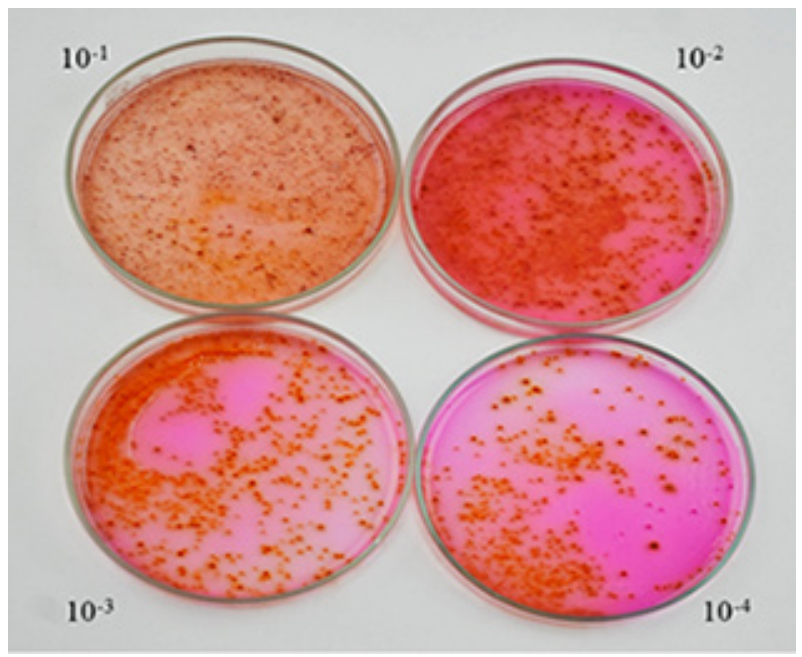

Fig 3. Pochonia chlamydosporia colonies in modified semi-selective medium after serial dilution.

to other substrates tested. Hidalgo et al. (2000) reported that rice can be used as a substrate for the mass production of $P$. chlamydosporia. Under in vitro conditions, isolation and quantification of colonies of $P$. chlamydosporia from various substrates using the modified medium were easier, faster and without any bacterial and fungal contamination.

Growth of $P$. chlamydosporia in soil inoculated 


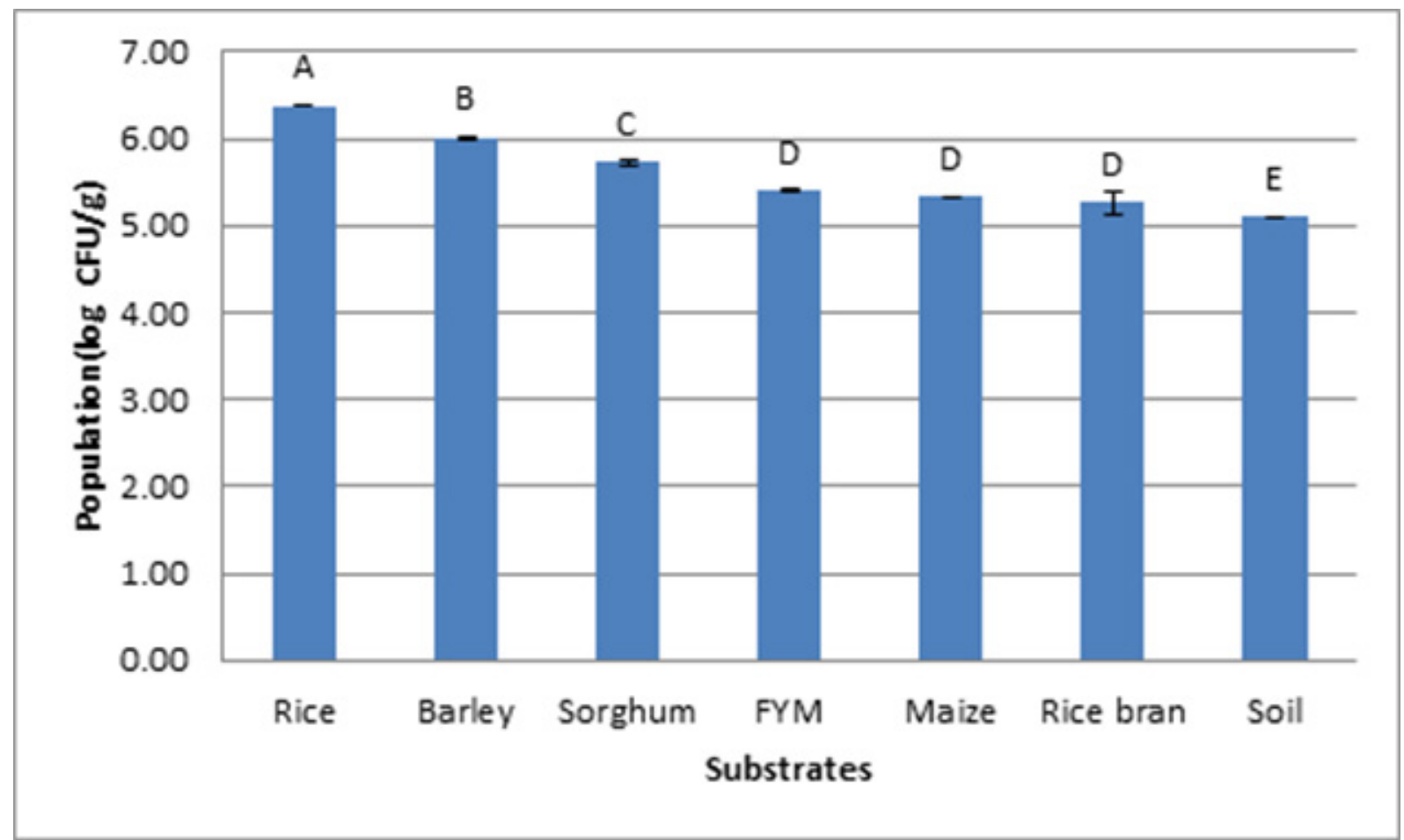

Fig 4. Quantification of Pochonia chlamydosporia on different solid substrates and soil 7 days after inoculation.

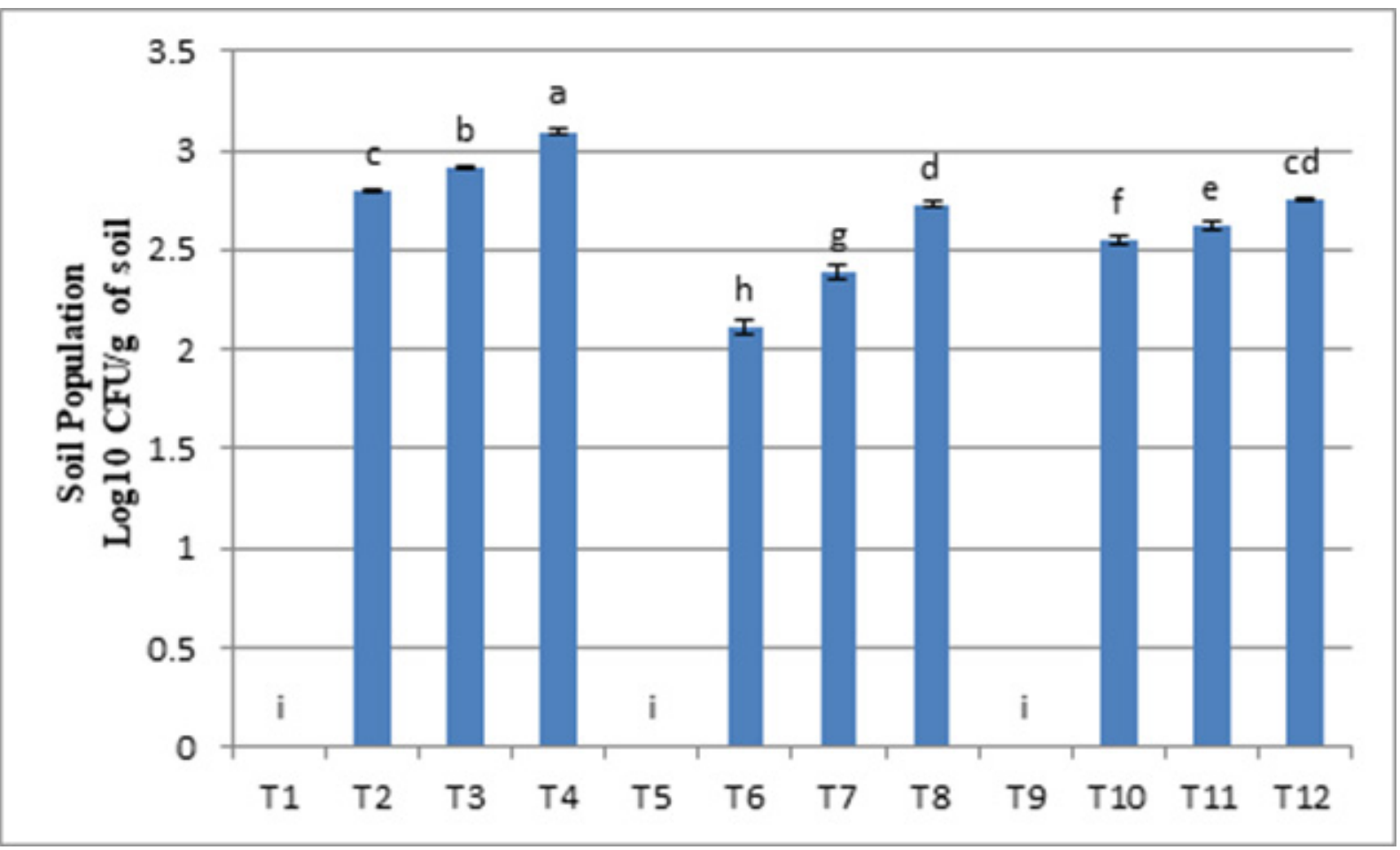

Fig 5. Quantification of Pochonia chlamydosporia $\left(10^{8} \mathrm{cfu} \mathrm{g}^{-1}\right)$ mass multiplied on rice grains in potting mixture 30 days after inoculation.

$\mathrm{T}_{1}$ : Uninoculated sterile soil, $\mathrm{T}_{2}$ : Sterile soil inoculated with $1 \mathrm{~g}^{*} \mathrm{~T}_{3}$ : Sterile soil inoculated with $3 \mathrm{~g}, \mathrm{~T}_{4}$ : Sterile soil inoculated with $5 \mathrm{~g}, \mathrm{~T}_{5}:$ Uninoculated non-sterile soil, $\mathrm{T}_{6}:$ Non sterile soil inoculated with $1 \mathrm{~g}$, $\mathrm{T}_{7}$ : Non sterile soil inoculated with $3 \mathrm{~g}, \mathrm{~T}_{8}$ : Non sterile soil inoculated with $5 \mathrm{~g}, \mathrm{~T}_{9}$ : Uninoculated vermiculite + farmyard manure, $\mathrm{T}_{10}$ : Vermiculite + farmyard manure inoculated with $1 \mathrm{~g}$, $\mathrm{T}_{11}$ : Vermiculite + farmyard manure inoculated with $3 \mathrm{~g}, \mathrm{~T}_{12}$ : Vermiculite + farmyard manure inoculated with $5 \mathrm{~g}$. 
with different doses of the fungus was also enumerated using the modified medium and the results are presented in Figure 5. The potting mixture inoculated with the fungus recorded significantly higher population one month after inoculation. The modified selective media was used for enumeration o and the medium was useful in estimating the population of $P$. chlamydosporia from non sterile soil samples without any fungal or bacterial contamination. The population of $P$. chlamydosporia was significantly high in treatments with soil than vermiculite + farmyard mixture. The population of $P$. chlamydosporia was found maximum in sterile soil inoculated with $5 \mathrm{~g}$ of inoculum.

The media developed was also useful in estimating the population of $P$. chlamydosporia isolated from rhizosphere soil samples without any contamination. In the present study, Kerry's semi-selective medium was modified for the efficient isolation and quantification of $P$. chlamydosporia from soils and other mass production substrates.

\section{Acknowledgments}

The senior author is thankful to Kerala State Council for Science, Technology and Environment (KSCSTE) No.24/FSHP/2016/ KSCSTE for funding. We thank Director and Head, Division of Crop Protection, ICARIISR, Kozhikode, for providing facilities and support; Mr. K Jayarajan, Senior Technical Officer, for the statistical analysis.

\section{References}

Aravind R, Eapen S J, Kumar A, Dinu A \& Ramana K V 2010 Screening of endophytic bacteria and evaluation of selected isolates for suppression of burrowing nematode (Radopholus similis Thorne) using three varieties of black pepper (Piper nigrum L.). Crop Prot. 29: 318-324.

Atkins S D, Hidalgo-Diaz L, Clark I M, Morton C O, De Oca N M, Gray P A \& Kerry B $R$ 2003a Approaches for monitoring the release of Pochonia chlamydosporia var. catenulata, a biocontrol agent of root-knot nematodes. Mycol. Res. 107: 206-212.
Atkins S D, Hidalgo-Diaz L, Kalisz H, Mauchline T H, Hirsch P R \& Kerry B R 2003b Development of a new management strategy for the control of root-knot nematodes (Meloidogyne spp.) in organic vegetable production. Pest Manag. Sci. 59: 183-189.

BourneJM, Kerry BR\&DeLeijFAA M1994Methods for the study of Verticillium chlamydosporium in the rhizosphere. J. Nematol. 26: 587-591.

Bruin G C A \& Edgington L V 1981 Adaptive resistance in Peronosporales to metalaxyl. Can. J. Plant Pathol. 3: 201-206.

Dong L Q \& Zhang K Q 2006 Microbial control of plant-parasitic nematodes: a five-party interaction. Plant Soil 288: 31-45.

Esteves I, Peteira B, Atkins S D, Magan N \& Kerry B 2009 Production of extracellular enzymes by different isolates of Pochonia chlamydosporia. Mycol. Res. 113: 867-876.

Fourie H, Ahuja P, Lammers J \& Daneel M 2016 Brassicacea-based management strategies as an alternative to combat nematode pests: A synopsis. Crop Prot. 80: 21-41.

Hidalgo-Díaz L, Bourne J M, Kerry B R \& Rodríguez M G 2000 Nematophagous Verticillium spp. in soils infested with Meloidogyne spp. in Cuba: isolation and screening. Int. J. Pest Manag. 46: 277-284.

Jacobs H, Gray S N \& Crump D H 2003 Interactions between nematophagous fungi and consequences for their potential as biological agents for the control of potato cyst nematodes. Mycol. Res. 107: 47-56.

Kerry B R 2000 Rhizosphere interactions and the exploitation of microbial agents for the biological control of plant-parasitic nematodes. Annu. Rev. Phytopathol. 38: 423441.

Kerry B R, Kirkwood I A, De Leij F A A M, Barba J, Leijdens M B \& Brookes P C 1993 Growth and survival of Verticillium chlamydosporium Goddard, a parasite of nematodes, in soil. Biocontrol Sci. Technol. 3: 355-365.

Manzanilla-López R H, Esteves I, Powers S J \& Kerry B R 2011. Effects of crop plants on abundance of Pochonia chlamydosporia and other fungal parasites of root-knot and 
potato cyst nematodes. Ann. Appl. Biol. 159: 118-129.

Manzanilla-López R H \& Lopez-Llorca L V (Eds.) 2017 Perspectives in Sustainable Nematode Management Through Pochonia chlamydosporia: Applications for Root and Rhizosphere Health. Springer. 411 pp.

Mauchline T H, Kerry B R \& Hirsch P R 2002 Quantification in soil and the rhizosphere of the nematophagous fungus Verticillium chlamydosporium by competitive PCR and comparison with selective plating. Appl. Environ. Microbiol. 68: 1846-1853.

Mauchline T H, Kerry B R \& Hirsch P R 2004 The biocontrol fungus Pochonia chlamydosporia shows nematode host preference at the infraspecific level. Mycol. Res. 108: 161-169.

Mensin S, Soytong K, McGovern R J \& Toanun C 2013 Effect of agricultural pesticides on the growth and sporulation of nematophagous fungi. J. Agric. Technol. 9: 953-961.
Morton C O, Mauchline T H, Kerry R \& Hirsch P R 2003 PCR-based DNA fingerprinting indicates host-related genetic variation in the nematophagous fungus Pochonia chlamydosporia. Mycol. Res. 107: 198-205.

Siddiqui I A, Atkins S D \& Kerry B R 2009 Relationship between saprotrophic growth in soil of different biotypes of Pochonia chlamydosporia and the infection of nematode eggs. Ann. Appl. Biol. 155: 131-141.

Sung G H, Hywel Jones N L, \& Sung J M 2007 Phylogenetic classification of Cordyceps and the clavicipitaceous fungi. Stud. Mycol. 57: 5-59.

Zare R, Gams W \& Evans H C 2001 A revision of Verticillium section Prostrata. V. The genus Pochonia, with notes on Rotiferophthora. Nova Hedwigia 73: 51-86. 